\title{
Imaging Coherent Acoustic Phonons in LaFeAsO with Ultrafast Electron Microscopy
}

\author{
Ryan A. Gnabasik ${ }^{1}$, Pranav K. Suri ${ }^{2}$, and David J. Flannigan ${ }^{1}$ \\ ${ }^{1}$ Department of Chemical Engineering and Materials Science, University of Minnesota, Minneapolis, \\ USA. \\ ${ }^{2}$ Department of Materials Science and Engineering, Drexel University, Philadelphia, USA.
}

Several iron pnictide materials, such as $\mathrm{LaFeAsO}$ and $\mathrm{BaFe}_{2} \mathrm{As}_{2}$, display high-temperature superconductivity when lightly doped [1]. Such compounds often undergo temperature-dependent structural, magnetic, and electronic transitions, which are generally thought to be linked in some way to the superconducting phase [2]. In order to resolve the interdependencies of the various degrees of freedom, ultrafast spectroscopic methods have frequently been used to determine timescales associated with charge-carrier and switching dynamics in the superconducting materials, and the more-recent advent of ultrafast structural probes (femtosecond X-ray and electron scattering) has led to insights into both the cuprates and the pnictides (both the doped and parent compounds) [3-6]. As with dedicated reciprocal-space methods, ultrafast transmission electron microscopy (UEM) provides a method for directly probing impulsively-excited structural dynamics, with the added benefit of real-space imaging with combined nanometer and sub-picosecond spatiotemporal resolutions [7].

Here we report the direct imaging of coherent acoustic-phonon dynamics in the 1111-type iron-pnictide parent compound LaFeAsO via UEM (Figure 1). Nucleation and launch of coherent and directional strain waves from the crystal edge following in situ femtosecond photoexcitation results in bright-field contrast modulation that is observed to propagate from the vacuum-crystal interface inward toward the thicker crystal regions. By quantifying the propagating contrast waves, phase-velocity dispersion and spatially-modulated frequency behaviors can be determined [Figure 1(c,d)]. Extending this approach to temperature-dependent measurements may enable determination of local, spatially-dependent elastic properties of the different structural phases (i.e., tetragonal and orthorhombic), and, potentially, provide information pertaining to nucleation and propagation of different phases as transitions progress. In addition, cross-correlation analysis of the picosecond-resolution images can be used to quantify perturbation of contrast features separate from the coherent acoustic-phonon wavefronts, providing additional insight into energy-relaxation pathways [8].

\section{References:}

[1] Y. Kamihara, et al., J. Am. Chem. Soc. 130 (2008), p. 3296.

[2] R. Fernandes, et al., Nature Phys. 10 (2014), p. 97.

[3] R. Mankowsky, et al., Nature 516 (2014), p. 71.

[4] M. Först, et al., Phys. Rev. Lett. 112 (2014), p. 157002.

[5] L. Rettig, et al., Phys. Rev. Lett. 114 (2015), p. 067402.

[6] H. Takahashi, et al., J. Phys. Soc. Jpn. 80 (2011), 013707.

[7] D. A. Plemmons, et al., Chem. Mater. 27 (2015), p. 3178.

[8] This material is based on work supported partially by the U.S. Department of Energy, Office of Science, Office of Basic Energy Sciences under Award No. DE-SC-0018204. This work was also supported partially by the National Science Foundation through the University of Minnesota 
MRSEC under Award No. DMR-1420013 and by the Arnold and Mabel Beckman Foundation through a 2015 Beckman Young Investigator Award.

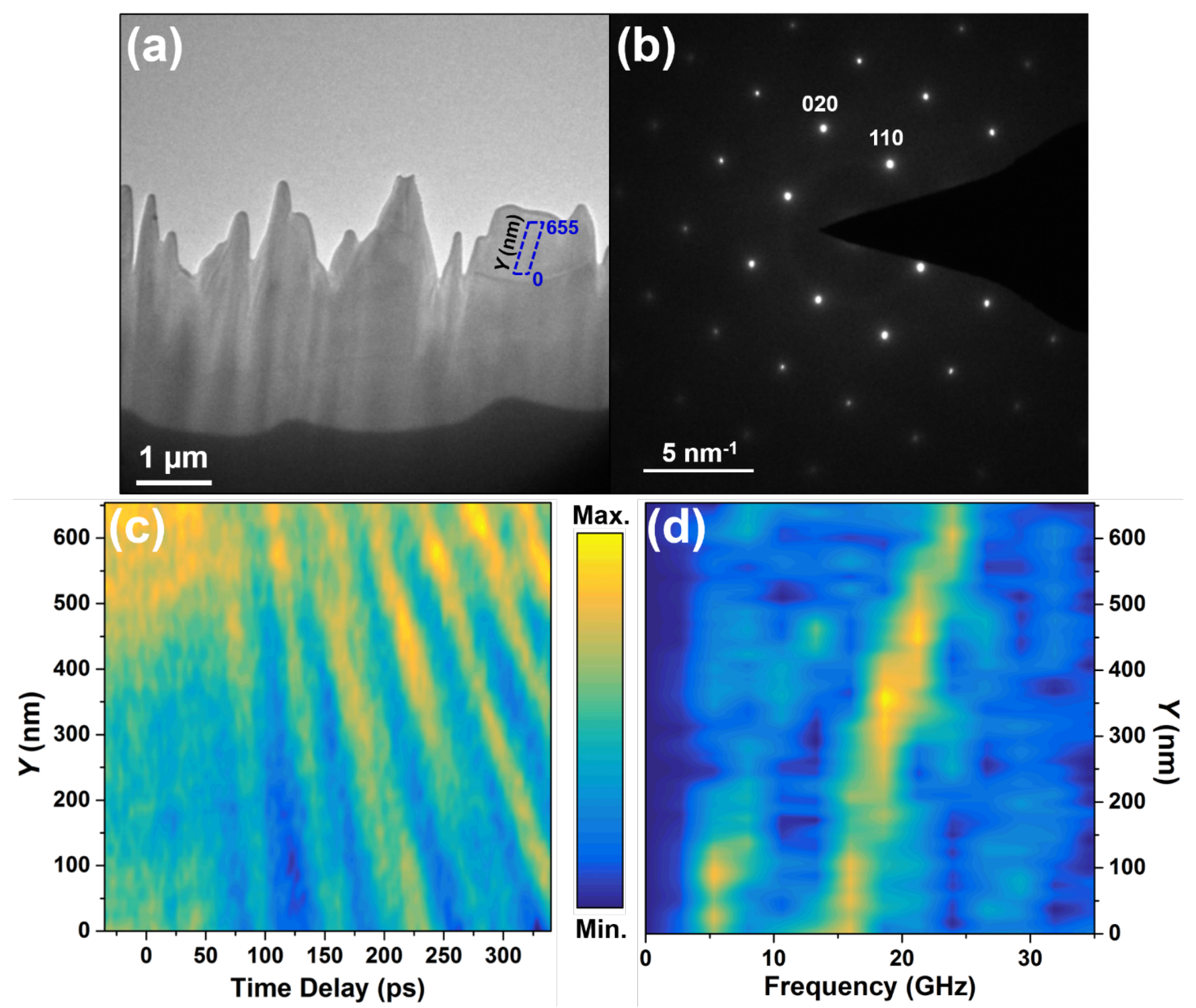

Figure 1. Coherent acoustic-phonon wavetrains in LaFeAsO at $300 \mathrm{~K}$. (a) Representative bright-field UEM image of an LaFeAsO specimen made using focused ion-beam milling. The dashed-blue parallelogram denotes the region of interest within which phonon dynamics were observed. The region spans from 0 to $655 \mathrm{~nm}$ [labeled $Y(\mathrm{~nm})$ ]. (b) Selected-area diffraction pattern from the specimen shown in (a), with the 020 and 110 Bragg spots indexed. (c) Space-time plot generated measured from the region indicated in (a), where the $y$-axis represents the vertical real-space distance along the analysis region; intensities of the pixels in the horizontal direction were averaged. Thus, the plot is comprised of single-pixel-wide contrast-profile snapshots in time. The bright (i.e., nearer to the Max. side of the color bar) bands represent contrast modulations propagating through the analysis region. (d) Fast Fourier transform (FFT) spatial map of the analysis region. The FFT was generated horizontally across the time axis for each real-space position measured in (c). 